\title{
The interannular bridge: A new technique for the management of tricuspid regurgitation in hypoplastic left heart syndrome
}

Kazuyoshi Kanno, MD, Akio Ikai, MD, PhD, Masaya Murata, MD, and Kisaburo Sakamoto, MD, Shizuoka, Japan

From the Department of Cardiovascular Surgery, Mt Fuji Shizuoka Children's Hospital, Shizuoka, Japan.

No specific funding was provided for this work.

Disclosures: Authors have nothing to disclose with regard to commercial support.

Authors have obtained the approval of the affiliated organization for this report.

Received for publication April 23, 2019; revisions received June 24, 2019; accepted for publication June 27, 2019; available ahead of print Aug 7, 2019

Address for reprints: Kazuyoshi Kanno, MD, Department of Cardiovascular Surgery, Mt Fuji Shizuoka Children's Hospital, 860 Urushiyama, Aoi-ku, Shizuoka, 420-8660, Japan (E-mail: kad-cib@umin.ac.jp).

J Thorac Cardiovasc Surg 2020;159:e219-21

$0022-5223 / \$ 36.00$

Copyright $(2019$ by The American Association for Thoracic Surgery

https://doi.org/10.1016/j.jtcvs.2019.06.080

- Video clip is available online.

For patients with hypoplastic left heart syndrome (HLHS), it is essential to control moderate to severe tricuspid regurgitation (TR) so that the Norwood procedure can be tolerated. Because of the vulnerability and future growth potential of the valve leaflet, it is difficult to adopt a conventional procedure for immobilizing the leaflets and annulus. We report here a case in which we used the interannular bridge technique to control central TR in an infant with HLHS who had undergone the Norwood procedure. This technique was able to improve coaptation without interfering with the subvalvular apparatus, the ability to control central regurgitation, and the preventive effects of interannular dilatation between the anterior and septal leaflets. The interannular bridge thus represents a new technique for tricuspid valvuloplasty for TR that can preserve valve function in patients with HLHS.

\section{CASE}

A female infant born at 39 weeks' gestation with a birth weight of $2675 \mathrm{~g}$ had HLHS (mitral atresia and aortic atresia) and moderate TR diagnosed on the basis of fetal echocardiography (Figure 1, A) and confirmed by postnatal echocardiography. According to our protocol, ${ }^{1}$ bilateral pulmonary artery banding was performed 2 days postpartum, and postoperative mild TR was observed. Patent ductus arteriosus was maintained by prostaglandin $\mathrm{E}_{1}$ infusion. At 26 days postpartum (body weight, $3008 \mathrm{~g}$ ), the

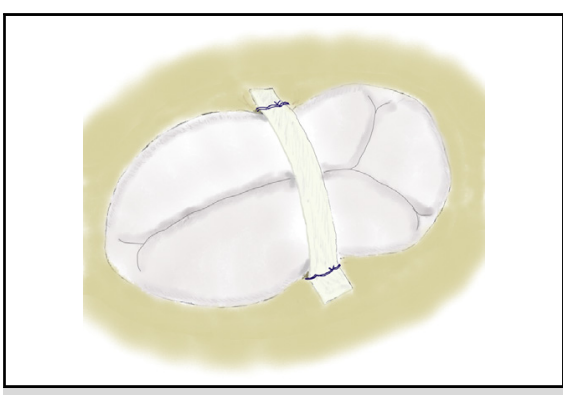

The interannular bridge technique improves central coaptation.

Central Message

The interannular bridge technique can control central regurgitation without any intervention to the subvalvular apparatus and can prevent the effects of annular dilatation.

See Commentaries on pages e 223 and e225.

Norwood procedure was performed; however, persistent moderate TR was noted (Figure 1, B).

Cardiopulmonary bypass was established by right brachiocephalic trunk and bicaval cannulations. The bilateral pulmonary artery banding was debanded. Atrial septal defect enlargement and tricuspid valvuloplasty were performed. Saline solution injection testing of the tricuspid valve on the beating heart and on the heart under cardiac arrest showed equally moderate regurgitation flow at the center of the valve coaptation. The posterior leaflet was more hypoplastic than the other 2 leaflets. After the septal and anterior leaflet clefts were closed, partial annuloplasty at both commissures of the posterior leaflet was performed to improve valve coaptation between the anterior and septal leaflets. Regurgitation flow decreased; however, we expected that this regurgitation would worsen with dilation of the annulus because of the response to an increase in saline solution injection volume and a history of TR after bilateral pulmonary artery banding. To prevent annular dilatation, we placed an interannular bridge between the annulus of the anterior and septal leaflets with an expanded polytetrafluoroethylene strip (width, $3 \mathrm{~mm}$; thickness, $0.4 \mathrm{~mm}$ ). We used a strip that was as long as possible to minimize regurgitation flow and sutured it to the atrial 


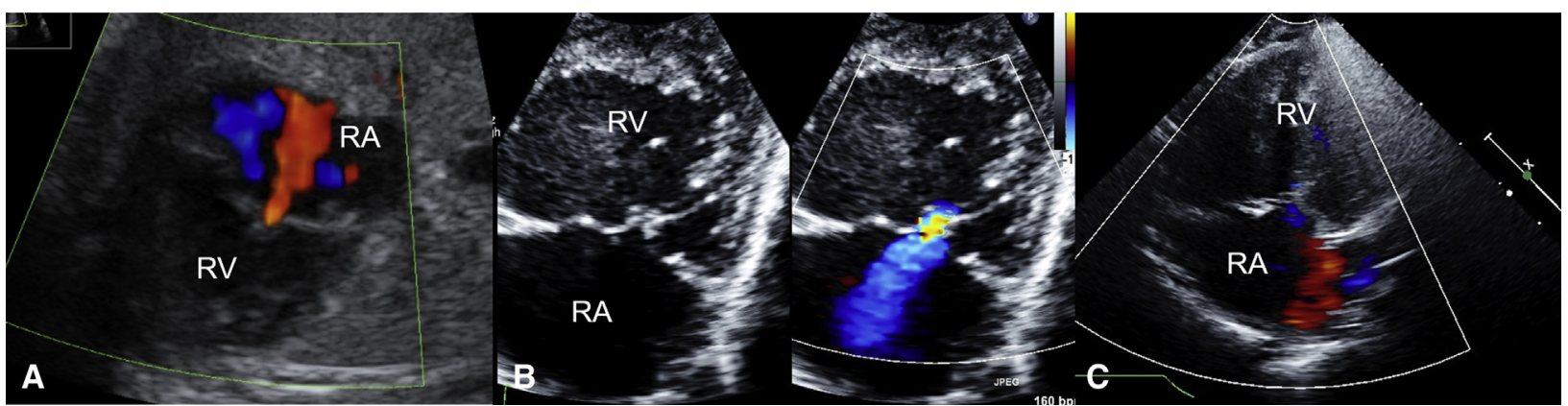

FIGURE 1. Findings of tricuspid regurgitation on echocardiography. A, Fetal echocardiography at 31 weeks' gestation. Moderate to severe tricuspid regurgitation was observed. B, Before the Norwood procedure. Moderate tricuspid regurgitation as a result of annular dilatation and shallow coaptation was observed. C, After the Norwood procedure. Improvements in coaptation and trivial tricuspid regurgitation were observed. $R A$, Right atrium; $R V$, right ventricle.

tissue just outside the annulus with 5-0 polypropylene suture (Figure 2 and Video 1).

The aortic arch was reconstructed, and a right ventriclepulmonary artery shunt was created. Weaning from cardiopulmonary bypass was successful, with only minor TR being noted. Delayed sternal closure was performed on postoperative day 6 .

The patient was discharged on day 69 . Echocardiography at discharge revealed trivial TR (Figure 1,C).
A bidirectional Glenn procedure was performed when the patient was 6 months of age. Preoperative echocardiography showed mild TR; cardiac catheterization showed a well-maintained cardiac function, with a right ventricular end-diastolic pressure of $8 \mathrm{~mm} \mathrm{Hg}$ and a right ventricular ejection fraction of $51.7 \%$.

An extracardiac conduit Fontan procedure with an 18$\mathrm{mm}$ expanded polytetrafluoroethylene graft was performed when the patient was 15 months of age. Preoperative and

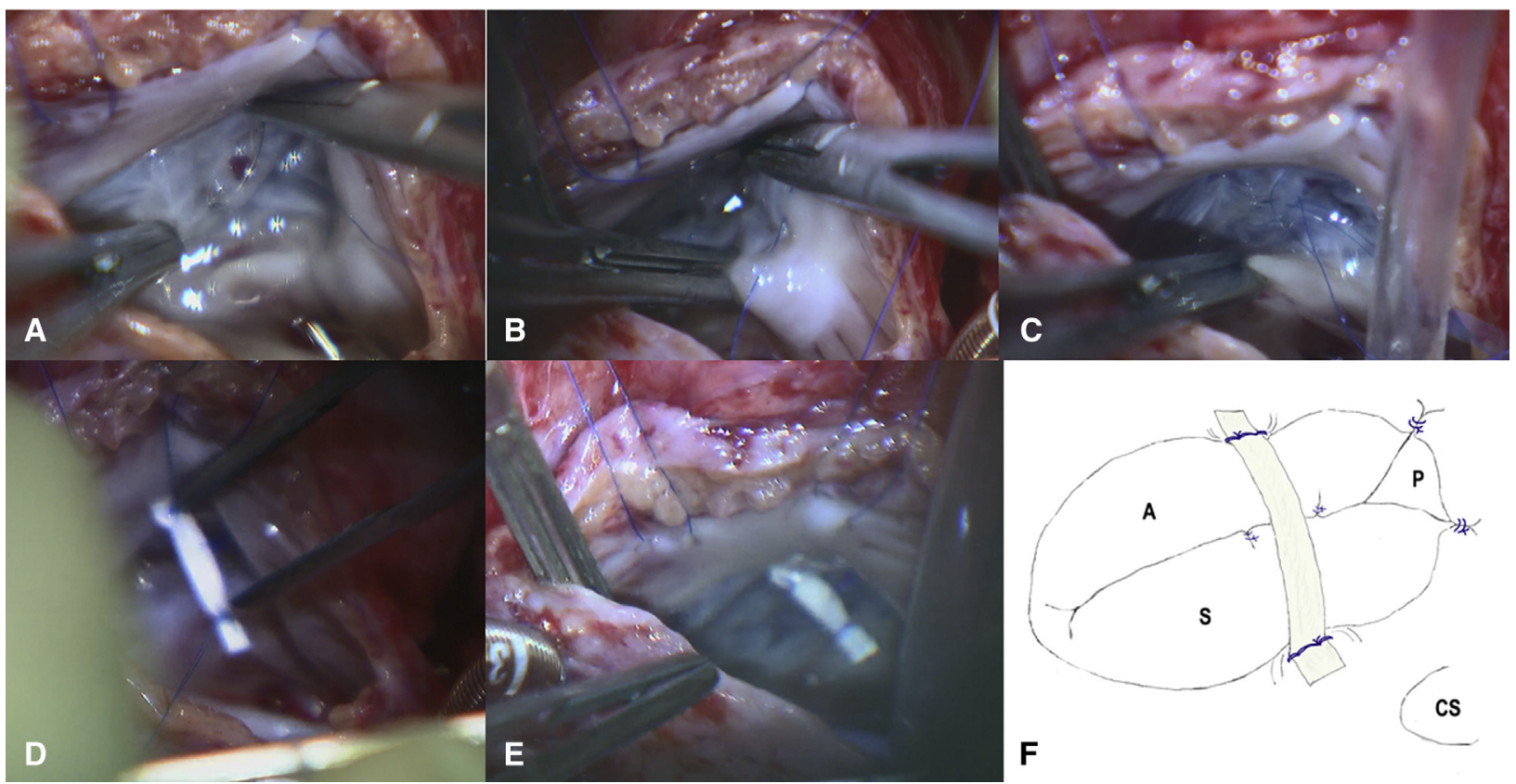

FIGURE 2. Overview of tricuspid valvuloplasty. A, Cleft closure was performed on the anterior and septal leaflets with 6-0 polypropylene suture. B, Commissuroplasty was performed on the anteroposterior and posteroseptal commissures with 5-0 polypropylene sutures. C, The good coaptation point was checked by moving the septal-side annulus with forceps during the saline solution injection test. D, The interannular bridge was installed. The expanded polytetrafluoroethylene strip (width, $3 \mathrm{~mm}$; thickness, $0.4 \mathrm{~mm}$ ) was stitched just outside (on the "atrial side") the annulus of the anterior and septal leaflets with 5-0 polypropylene sutures. E, No regurgitation was observed during the saline solution injection test. F, Schema. A, Anterior leaflet; $P$, posterior leaflet; $S$, septal leaflet; $C S$, coronary sinus. 


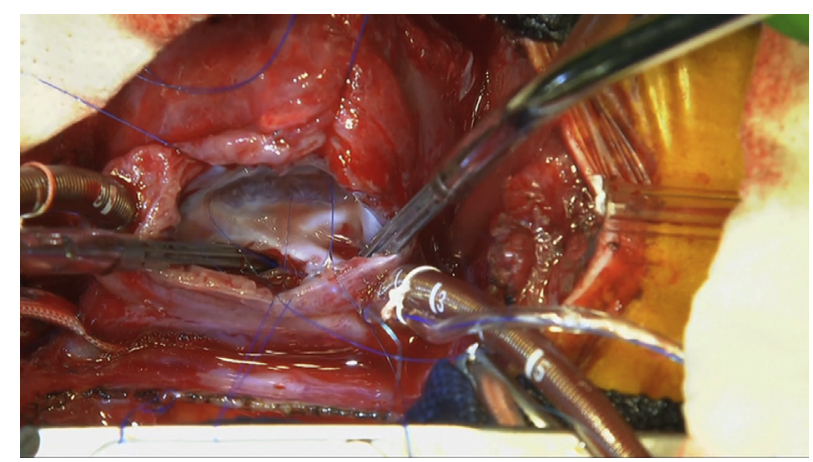

VIDEO 1. This video clip demonstrates the tricuspid valvuloplasty. Saline solution injection testing of the tricuspid valve showed moderate regurgitation flow at the center of the valve coaptation. The suture that secures the anterior leaflet and septal leaflet was temporarily placed to reproduce coaptation of the leaflets. This suture was eventually removed. The septal and anterior leaflet clefts were closed. The partial annuloplasty at bilateral commissures of the posterior leaflet was performed. Regurgitant flow decreased as a result of an improvement of valve coaptation between the anterior and septal leaflets; however, this regurgitation worsened with dilation of the annulus in response to an increase in saline solution injection volume. To prevent annular dilatation, the inter-annular bridge was placed. Video available at: https://www.jtcvs.org/article/S0022-5223(19)31399-6/ fulltext.

postoperative echocardiography showed mild TR, with no evidence of stenosis. Cardiac catheterization performed 1 year after the Fontan procedure showed a right ventricular end-diastolic pressure of $5 \mathrm{~mm} \mathrm{Hg}$, a right ventricular ejection fraction of $54.1 \%$, and a Fontan pressure of $13 \mathrm{~mm} \mathrm{Hg}$. Thirty months after the Fontan procedure, echocardiography revealed mild TR with no evidence of stenosis.

\section{DISCUSSION}

For patients with HLHS, it is essential to control moderate to severe TR so that the Norwood procedure can be tolerated. Considering the vulnerability and future growth potential of the valve leaflet, especially at the time of the Norwood procedure, it is difficult to adopt a conventional procedure for immobilizing the leaflets and annulus. Ohye and colleagues ${ }^{2}$ reported that patients requiring a combined procedure beyond partial annuloplasty did not obtain significant regurgitation control. Similarly, we found worsened regurgitation after tricuspid valvuloplasty. In HLHS, right ventricle and tricuspid annulus morphologies are different from those of normal hearts, and interactions between the tricuspid valve and the right ventricle are abnormal, making it easier to create a coaptation gap in the center of the valve. ${ }^{3}$ Bautista-Hernandez and coworkers ${ }^{4}$ noted annular dilatation and anterior leaflet prolapse as the main factors for TR in HLHS; they mentioned valvuloplasty with movement of the anterior papillary muscle. In our case, regurgitation from the center persisted after the use of such conventional techniques as commissuroplasty, cleft closure, and partial annuloplasty.

We therefore used an interannular bridge technique to control central regurgitation. This was advantageous because of its ability to improve coaptation without interfering with the subvalvular apparatus, the ability to control central regurgitation, and the preventive effects of interannular dilatation between the anterior and septal leaflets. A bridgelike procedure for common atrioventricular valves has been previously reported, ${ }^{5}$ but our procedure has important differences. A feature of our procedure is that the bridge is not fixed anywhere in the valve structure. Under the bridge, the leaflets remain nearly normal, and the opening is not split in two. The long-term impact of the bridging on tricuspid valve morphology is unknown; however, this patient did not present with any findings of stenosis for 45 months after tricuspid valvuloplasty. The interannular bridge thus represents a new technique for tricuspid valvuloplasty that can preserve the valve function for patients with TR in HLHS.

\section{References}

1. Ota N, Murata M, Tosaka Y, Ide Y, Tachi M, Ito H, et al. Is routine rapid-staged bilateral pulmonary artery banding before stage 1 Norwood a viable strategy? J Thorac Cardiovasc Surg. 2014;148:1519-25.

2. Ohye RG, Gomez CA, Goldberg CS, Graves HL, Devaney EJ, Bove EL. Tricuspid valve repair in hypoplastic left heart syndrome. J Thorac Cardiovasc Surg. 2004; 127:465-72.

3. Nii M, Guerra V, Roman KS, Macgowan CK, Smallhorn JF. Three-dimensional tricuspid annular function provides insight into the mechanisms of tricuspid valve regurgitation in classic hypoplastic left heart syndrome. J Am Soc Echocardiogr. 2006;19:391-402.

4. Bautista-Hernandez V, Brown DW, Loyola H, Myers PO, Borisuk M, del Nido PJ, et al. Mechanisms of tricuspid regurgitation in patients with hypoplastic left heart syndrome undergoing tricuspid valvuloplasty. J Thorac Cardiovasc Surg. 2014; 148:832-8; discussion 838-40.

5. Sughimoto K, Konstantinov IE, Brizard CP, d'Udekem Y. Polytetrafluoroethylene bridge for atrioventricular valve repair in single-ventricle palliation. J Thorac Cardiovasc Surg. 2015;149:641-3. 